\title{
Femtosecond Laser-Assisted Refractive Autokeratoplasty: A Pilot Study
}

\author{
Halina Viktorovna Sitnik ${ }^{1 *}$, Aleksey Yurevich Slonimsky², Yuriy Borisovich Slonimsky³, \\ Tatsiana Aleksandrovna Imshenetskaya ${ }^{1}$ \\ ${ }^{1}$ Belarusian Medical Academy of Post-Graduate Education, Minsk, Belarus \\ ${ }^{2}$ State Budget Institution of Health "City Clinical Hospital Botkin Moscow", Ophthalmic Hospital, Moscow, \\ Russia \\ ${ }^{3}$ Russian Medical Academy of Post-Graduate Education, Moscow, Russia \\ Email: "sitnik_halina@mail.ru
}

Received 10 February 2016; accepted 9 May 2016; published 12 May 2016

Copyright (C) 2016 by authors and Scientific Research Publishing Inc.

This work is licensed under the Creative Commons Attribution International License (CC BY). http://creativecommons.org/licenses/by/4.0/

(c) (i) Open Access

\section{Abstract}

Purpose: To develop a new method of femtosecond laser-assisted refractive autokeratoplasty (FRAK) in advanced keratoconus and to evaluate preliminarily early clinical results. Methods: A total of 17 patients with stable advanced keratoconus and a mean age of $33 \pm 8.4$ years were included in the study. FRAK was performed in all cases with the IntraLase $60 \mathrm{kHz}$ (Abbott Medical Optics Inc.). A 2-step resection of corneal stroma was performed using the femtosecond laser, with the generation of a circular corneal flap with wedge-shaped profile. After flap removal, the corneal wound was sutured. Results: The surgical procedure and early postoperative period were uneventful in all cases. Mean uncorrected distance visual acuity (UDVA) improved significantly from $0.07 \pm 0.03$ preoperatively to $0.26 \pm 0.13$ at 3 months after surgery. Improvement in corrected distance visual acuity (CDVA) was observed in $94.1 \%$ of cases, with $76.5 \%$ of eyes showing an improvement of more than 3 lines. Between 3 and 6 months after surgery, an additional improvement was observed in UDVA and CDVA. Corneal cylinder decreased significantly from $9.1 \pm 3.8 \mathrm{D}$ preoperatively to $4.4 \pm 2.75 \mathrm{D}$ at 6 months postoperatively. Conclusions: FRAK may be an alternative treatment in stable advanced keratoconus, allowing a significant visual improvement and corneal regularization while saving the patient's own corneal tissue. The non-penetrating nature of the surgical technique helps to minimize the risks associated to this type of surgery. Further research is needed to determine the functional long-term outcomes.

\section{Keywords}

Keratoconus, Keratoplasty, Autokeratoplasty, Femtosecond Laser

\footnotetext{
${ }^{*}$ Corresponding author.
}

How to cite this paper: Sitnik, H.V., Slonimsky, A.Y., Slonimsky, Y.B. and Imshenetskaya, T.A. (2016) Femtosecond Laser-Assisted Refractive Autokeratoplasty: A Pilot Study. Open Journal of Ophthalmology, 6, 86-93. 


\section{Introduction}

Keratoconus is a usually bilateral corneal disorder that clinically manifests in form of a progressive corneal conic protrusion and thinning, with an associated visual deterioration due to the induction of corneal irregular astigmatism [1] [2]. The incidence of keratoconus varies broadly among different ethnic groups, with reported values of 57 patients per 100,000 people in the Caucasian population and 229 per 100,000 people in Asia [1]-[3]. The exact aetiology and pathogenesis of keratoconus are still not completely understood. The incidence seems to be higher in males [1]-[4].

Several options have been described for the management and visual rehabilitation in keratoconus, such as spectacle correction, contact lenses, implantation of intracorneal ring segments, corneal collagen cross-linking, topography-guided custom ablation and lamellar or penetrating keratoplasty [5]. In cases of advanced keratoconus (stages III and IV according to the Amsler grading system) [6], there is a significant deterioration of the visual function with low or no improvement and tolerance with spectacles or contact lenses [6]. In such cases, a surgical option is required for obtaining an acceptable visual improvement and avoiding the progression of the disease [7].

Corneal collagen cross-linking is based on the photopolymerization of corneal collagen fibres after activating the photosensibilizer riboflavin by using ultraviolet radiation. This leads to a change of the mechanical properties of the cornea. Long-term studies have shown that a higher efficacy of this therapy for stabilization and improvement of topographic parameters and visual acuity is achieved for stages I or II of keratoconus [8] [9]. The implantation of intracorneal ring segments has shown its best functional results in keratoconus stages II and III [10]. In stages III and IV of keratoconus, deep anterior lamellar keratoplasty is considered an efficient treatment option that preserves patient's own Descemet membrane and endothelium, resulting consequently in a significant reduction of the graft rejection rate [11]-[13]. Penetrating keratoplasty should only be considered in cases of rough scars and ruptures of Descemet's membrane [14] [15].

The application of femtosecond laser-based technology in ophthalmology in the last decade has undoubtedly opened new possibilities in the treatment of patients with corneal pathologies [16], contributing specifically to improved functional results after corneal transplantation [12]. Femtosecond lasers allow corneal tissue cuts with a selected profile as well as high accuracy and reproducibility, providing at the same time the possibility of an individual approach to surgical planning [12] [17]-[19]. The purpose of the current study was to develop a new method of refractive autokeratoplasty with the use of the femtosecond laser technology for the management of patients with advanced keratoconus and to evaluate the early clinical outcomes of this treatment.

\section{Methods}

\subsection{Patients}

This study enrolled 17 consecutive patients (15 men, 2 women) with advanced keratoconus and a mean age of $33.0 \pm 8.4$ years (median: 31 years). All patients underwent femtosecond-based refractive autokeratoplasty (FRAK) using the IntraLase platform (Abbott Medical Optics Inc.) in the period from October 2014 to June 2015 in the Municipal Clinical Hospital No. 10 in Minsk. The inclusion in the study and the surgical procedure were performed after the patient was informed about the nature of the research and the risks of the surgical procedure. Likewise, all patients signed an informed consent form in accordance with the tenets of the Declaration of Helsinki.

The inclusion criteria for this study were: stage III-IV keratoconus according to the Amsler-Krumeich grading system, no registration of keratoconus progression during the last 1.5 years, corneal thickness at the cone apex of $330 \mu \mathrm{m}$ or more, corneal thickness in the zone of $7-10 \mathrm{~mm}$ of $500 \mu \mathrm{m}$ or more, anterior chamber depth of more than $3.5 \mathrm{~mm}$, open anterior chamber angle, normal level of intraocular pressure, low tolerance to spectacle or contact lens refractive correction, and absence of Descemet's membrane rupture or severe stromal opacity. Rigid gas permeable contact lenses were used by 4 patients of our sample, with only one of them wearing them 6 to 8 hours per day. The other 3 patients wearing contact lenses reported low tolerance, with the impossibility of a comfortable wearing of more than 2 to 4 hours a day. The fitting of rigid gas permeable contact lenses was not possible in the remaining 13 patients.

Statistical analysis was performed with SPSS (Statistical Package for Social Sciences) software version 17. Wilcoxon and $t$ tests were used for data comparison. Any p value less than $0.05(p<0.05)$ was considered as significant. 


\subsection{Examination Protocol}

All patients underwent a complete ophthalmological examination before surgery as well as during the entire follow-up period. The following tests were included: measurement of uncorrected (UDVA) and corrected distance visual acuity (CDVA), manifest refraction, pneumotonometry (Topcon, Japan), biomicroscopy, keratometry and pachymetry (TMS-5, Tomey, Japan), optical coherence tomography evaluation of the anterior segment (Visante OCT, Carl Zeiss Meditec), and corneal endothelial microscopy with estimation of the endothelial cell density (Topcon SP-3000P, Japan). The postoperative follow-up in the patients of our series ranged from 2 to 8 months, with controlled postoperative examinations at 1,3 and 6 months after surgery.

\subsection{Surgical Procedure}

All surgeries were performed under local anaesthesia by the same experienced surgeon (HVS). Before surgery, antibiotic drops (fluoroquinolones) were prescribed to 6 to 12 hours before operation. The femtosecond laser IntraLase $60 \mathrm{kHz}$ (program Keratoplasty) was used in all cases to perform the corneal cuts with the predicted depth and angle. The crucial calculation of the parameters defining the cut was performed individually, based on pachymetric and topographic data. The width of the corneal ectatic area and its location relative to the visual axis were considered for the selection of the cut diameter. The depth of the cut was $90 \%$ of mean corneal thickness in the area where the cut was planned to be performed. After programming the surgical parameters, the central 6-mm corneal zone was marked with the use of a corneal marker in order to facilitate the subsequent laser centration. Then, after obtaining the adequate corneal applanation using the vacuum ring and disposable interface, the first circular corneal cut was performed along the ectasia zone (not less than $8.0 \mathrm{~mm}$ in diameter), with an angle calculated individually for a depth of up to $90 \%$ of corneal stroma thickness in this area. After this, a second circular corneal cut was performed perpendicular to the surface, with a separation from the first one ranging from 150 to $300 \mathrm{~nm}$. Both cuts intersected at the planned depth, creating a circular corneal flap with a wedge-shaped profile. Then, the vacuum fixator was removed. Figure 1(A) shows the status of the cornea immediately after the creation of the wedge-shaped flap with the femtosecond laser.

After the dissection of the flap, the patient was placed under the surgical microscope where the second part of the surgical procedure was performed. If necessary, the cornea was marked again with a surgical marker or corneal suture marker to obtain a more precise approximation of wound margins. The corneal flap was easily removed using the forceps and spatula (Figures 1(B)-(D)) while controlling the integrity of the corneal wound and the absence of signs of anterior chamber perforation (intraocular fluid filtration). In some cases, a paracentesis was performed for reducing the anterior chamber depth and facilitating the adaptation of the wound margins. After these steps, the first four cardinal interrupted sutures were placed on the corneal wound margins (Nylon 10-0) with radial orientation in the 3, 6, 9 and 12 hour clock meridians (Figure 1(E)). The final number of required sutures was defined individually in each case, with the general criterion of achieving a satisfactory approximation of corneal wound margins. In the majority of cases, the number of interrupted buried sutures ranged from 8 to 12 (Figure 1(F)). A postoperative pharmacological therapy was applied in all cases: antibiotic drops (Oftaquix, Santen) 5 times daily for 3 days and 4 times daily afterwards for up to 7 days; dexamethasone $0.1 \%$ drops 4 times daily for 10 days, 3 times daily for up to 1 month after the operation and 2 times daily afterwards; pantothenic acid 5\% (Corneregel, Bausch \& Lomb) every 3 hours for 3 days and then 4 times daily. At 1 week postoperatively, this therapy was complemented with tear substitutes (Systane-Ultra, Alcon).

The principal idea of this surgical technique was to achieve a corneal surface flattening and consequently a visual improvement. For this reason, a two-stage resection of corneal stroma was performed with the use of a femtosecond laser, followed by the insertion of corneal sutures.

\section{Results}

Preoperatively, all patients had myopic refraction and myopic astigmatism. The diagnosis of keratoconus had been performed from 4 to 14 years before our preoperative examination. A total of 3 and 14 patients had a stage III and IV keratoconus, respectively. A keratoconus of the same stage was present in both eyes of the same patient in 9 cases. Corneal collagen crosslinking had been previously performed in 4 patients between 1.4 to 4 years before our preoperative examination. In the remaining cases, crosslinking had not been done due to the seriously advanced stage of the disease. In 2 patients, penetrating keratoplasty had been performed in the right eye, with a high level of remaining postoperative astigmatism (9.5 and $12 \mathrm{D})$. Mean preoperative decimal uncor- 


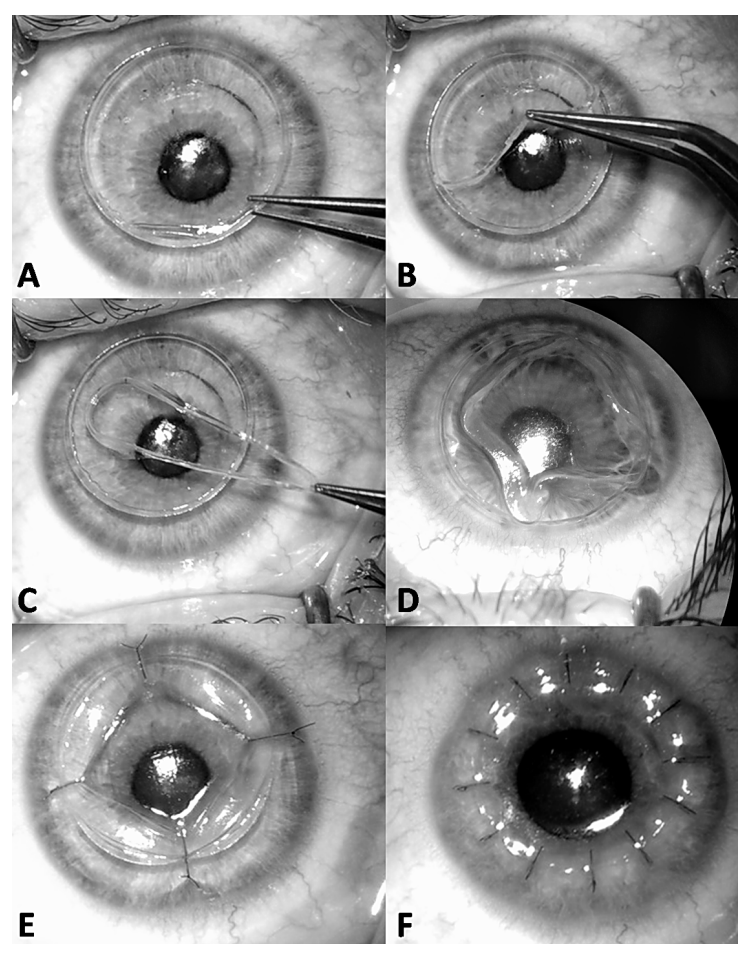

Figure 1. Stages of femtosecond laser-guided refractive autokeratoplasty. (A) Creation of the wedge-shaped flap with the femtosecond laser. (B) Lifting of the corneal flap created. (C) Removal of the corneal flap. (D) Placement of the removed flap over the residual cornea. (E) Placement of the first four cardinal interrupted sutures on the corneal wound margins (Nylon 10-0) with radial orientation in the 3, 6, 9 and 12 clock hour meridians. (F) Final suturing until achieving a satisfactory approximation of corneal wound margins.

Table 1. Preoperative pachymetric and corneal topographic data.

\begin{tabular}{|c|c|c|c|c|c|}
\hline \multirow{2}{*}{ Keratoconus stage } & \multirow{2}{*}{$\begin{array}{c}\text { Minimum corneal } \\
\text { thickness } \\
(\mu \mathrm{m})\end{array}$} & \multirow{2}{*}{$\begin{array}{l}\text { Corneal thickness in the } \\
7 \text { to } 10-\mathrm{mm} \text { zone } \\
(\mu \mathrm{m})\end{array}$} & \multicolumn{3}{|c|}{ Topographic indices } \\
\hline & & & $K 1(D)$ & $K 2(D)$ & Astigmatism (D) \\
\hline $\begin{array}{c}\text { III } \\
\text { TMS-5, Tomey } \\
\text { Visante OCT, CZM }\end{array}$ & $\begin{array}{l}393 \pm 24.8 \\
365 \pm 18.8\end{array}$ & $\begin{array}{c}537.4 \pm 24.6 \\
525.43 \pm 23.7\end{array}$ & $54.8 \pm 0.9$ & $48.9 \pm 0.1$ & $4.0 \pm 0.7$ \\
\hline $\begin{array}{c}\text { IV } \\
\text { TMS-5, Tomey } \\
\text { Visante OCT, CZM }\end{array}$ & $\begin{array}{l}371 \pm 23.8 \\
355 \pm 16.4\end{array}$ & $\begin{array}{c}544.6 \pm 23.6 \\
530.44 \pm 21.8\end{array}$ & $59.8 \pm 5.7$ & $54.8 \pm 5.8$ & $8.1 \pm 3.8$ \\
\hline
\end{tabular}

"Abbreviations: D, diopters; K1, curvature in the steepest keratometric meridian in the 3-mm central zone; K2, curvature in the flattest keratometric meridian in the 3-mm central zone.

rected (UDVA) and corrected distance visual acuity (CDVA) in our sample were 0.07 and 0.15 , respectively.

Table 1 summarizes the preoperative pachymetric and corneal topographic data in the analysed sample. Comparing the pachymetric data obtained with the TMS-5 (Tomey, Japan) and Visante OCT (Carl Zeiss Meditec) systems, a difference from 35 to $57 \mu \mathrm{m}$ was detected in the same corneal meridians, with higher values of the TMS-5 system. For safety purposes, the OCT pachymetric data were used for the calculation of the cut parameters with the femtosecond laser. Mean preoperative anterior chamber depth (ACD) in keratoconus eyes stage III and IV was $3.61 \pm 0.15$ and $3.78 \pm 0.17 \mathrm{~mm}$, respectively.

\subsection{Visual and Refractive Changes}

Postoperative follow-up ranged from 3 to 10 months. Figure 2 summarizes the changes in UDVA and CDVA after the surgery. Mean decimal UDVA improved significantly from $0.07 \pm 0.03$ preoperatively to $0.26 \pm 0.13$ at 3 months after surgery $(\mathrm{p}<0.01)$. An improvement in CDVA was observed in $94.1 \%$ of cases, with $76.5 \%$ im- 
proving more than 3 lines. In the postoperative period from 3 to 6 months, no significant changes were detected in UDVA and CDVA ( $>$ > 0.05).

\subsection{Topographic, Pachymetric and Anterior Segment Changes}

Maximum keratometry changed from a mean preoperative value of $60.1 \pm 5.7 \mathrm{D}$ to a 3-month postoperative value of $53.2 \pm 3.8 \mathrm{D}(\mathrm{p}<0.01)$, whereas minimum keratometry decreased from $54.8 \pm 5.8 \mathrm{D}$ preoperatively to $46.4 \pm 3.6 \mathrm{D}$ postoperatively ( $<0.01$ ). Likewise, the magnitude of corneal astigmatism decreased significantly from $9.1 \pm 3.8$ preoperatively to $4.4 \pm 2.75 \mathrm{D}$ postoperatively $(\mathrm{p}<0.01)$. An 8 -month follow-up was completed by 4 patients, with no significant changes during the postoperative period in the magnitude of keratometric indices $(\mathrm{p}>0.05)$.

The thinnest pachymetric data did not show significant differences with respect to preoperative values at 1,3 and 6 months postoperatively ( $\mathrm{p}>0.05$ ).

Figure 3 shows the status of the anterior chamber at the slit lamp examination on the first postoperative day in two different eyes of our series. A gradual restoration of ACD was observed in the first postoperative month, with a mean value of $2.9 \pm 0.26 \mathrm{~mm}$ at this time point. No significant changes were observed in ACD between 3 and 6 months after surgery, with a mean 6-month postoperative value of $3.10 \pm 0.23 \mathrm{~mm}$ (Figure 4).

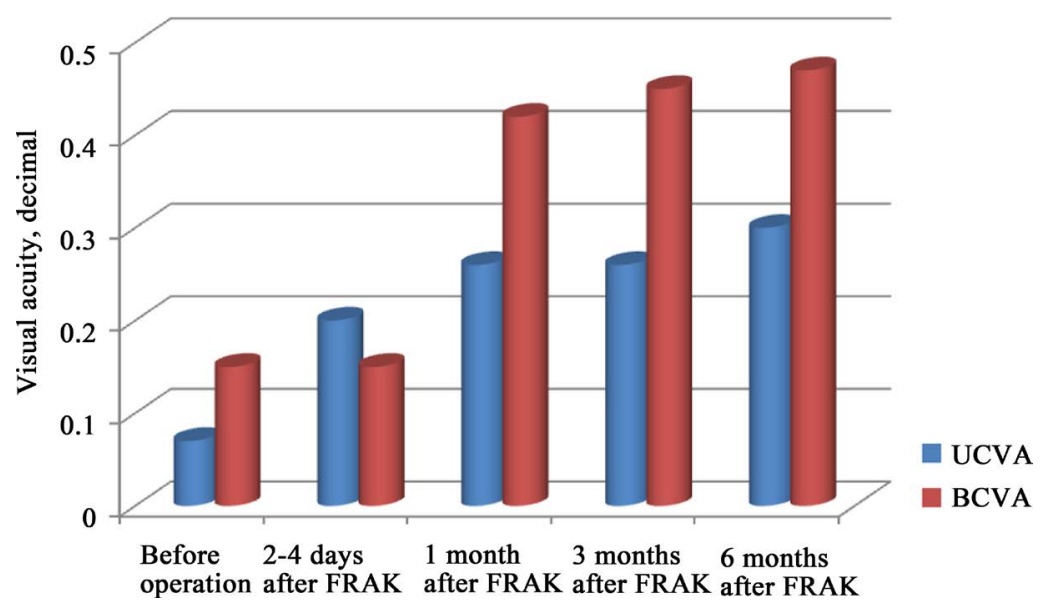

Figure 2. Changes in mean uncorrected (UDVA) and corrected distance visual acuity (CDVA) after femtosecond-guided refractive autokeratoplasty (FRAK).

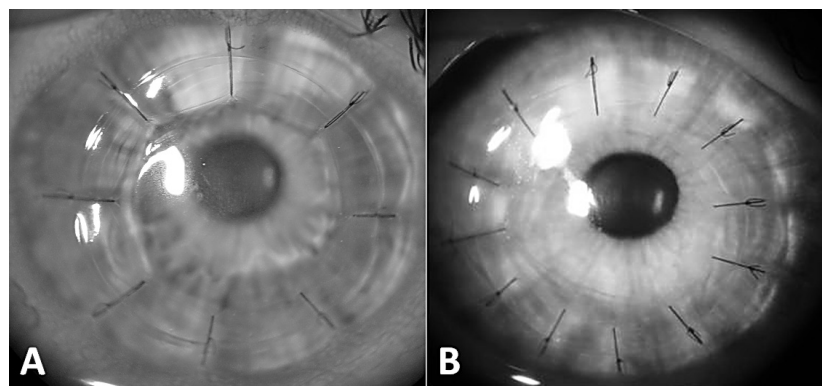

Figure 3. Status of the anterior eye segment on slit lamp examination on the first postoperative day in two different eyes of our series. A. Case with 8 interrupted buried sutures. B. Case with 12 interrupted buried sutures.

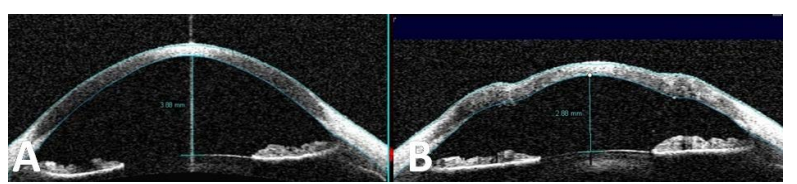

Figure 4. Changes in the anterior chamber depth of a female patient undergoing FRAK before surgery and 5 days postoperatively. Anterior chamber depth changed from $4.0 \mathrm{~mm}$ preoperatively to $2.81 \mathrm{~mm}$ postoperatively. 


\subsection{Corneal Endothelial and Intraocular Pressure (IOP) Changes}

Preoperative corneal endothelial cell density could not be measured in 15 of 17 cases due to the presence of a marked conical corneal deformation. At 1 month after surgery, mean endothelial cell density in the central corneal zone was $2592.0 \pm 120.2 \mathrm{cell} / \mathrm{mm}^{2}$. No significant changes in cell density were observed in the postoperative period from 3 to 6 months after surgery $(p>0.05$ ), with a mean 6-month postoperative value of $2569.0 \pm$ $113.8 \mathrm{cell} / \mathrm{mm}^{2}$. IOP did not increase significantly in any case, with a mean 6-month postoperative value of 16.0 $\pm 2.3 \mathrm{~mm} \mathrm{Hg}$.

\subsection{Complications}

No suture-related complications were observed during the follow-up period (wound dehiscence, sagging, neovascularization). Corneal suture removal was planned at 10 to 12 months after surgery. Due to the application of a vacuum fixator at the stage of corneal applanation, subconjunctival hemorrhage was present in some cases in the early postoperative period, but resolved spontaneously within few days. All patients were informed about this potential condition beforehand. Complaints of photophobia and tearing of different severity were reported by most of the patients in the first 2 to 3 days after surgery. All patients reported a subjective significant improvement of visual quality in the operated eye at 3 to 5 days postoperatively.

\section{Discussion}

Corneal transplantation should be considered as one of the last options in keratoconus management. Clinical guidelines for this disease have changed significantly in the last decade, being focused now on preservation of patient's own tissue and on the selection of the least invasive treatment if possible [20]. Deep anterior lamellar corneal transplantation has almost completely substituted penetrating keratoplasty and is considered to be the "gold standard" treatment in advanced stages of keratoconus [7]. Despite the favourable conditions for the development of keratoplasty techniques in many countries, the deficit of corneal donor tissues is an important handicap. For this reason, the option of autokeratoplasty has been developed and tested in some conditions [21]-[23]. The main benefits of autokeratoplasty are the retention of host endothelium, thereby eliminating both the risk of endothelial rejection and the prolonged attrition of endothelial cell numbers that occur following penetrating keratoplasty, and the reduced requirement for postoperative steroid therapy with its associated complications [24]. All these issues have been considered for the development of a new treatment method for advanced keratoconus.

Patients with advanced keratoconus are commonly young, with high visual requirements and expectations as well as the need for a fast visual rehabilitation and reduction of disability. In stable stages of the disease, the preservation of corneal thickness and transparency is crucial at the same time that the optical properties of the patient's own cornea are modified for improving visual acuity and quality of life. This has been attempted with corneal collagen crosslinking [25] and implantation of intracorneal ring segments [26], with very limited or unsuccessful outcomes. The application and implementation of femtosecond laser technology in ocular surgery deliver new opportunities regarding accuracy, programmability and predictability of surgical procedures, as well as an individual approach at the stage of planning and performing the operation [16]. We described a new method of treatment for patients with advanced keratoconus using the femtosecond laser technology, the femtosecond-guided refractive autokeratoplasty (FRAK). To this date, only minimal evidence of the potential usefulness of manual autokeratoplasty has been reported for keratoconus patients [27] [28]. Likewise, the current study was also aimed at reporting the first clinical result with this new technique.

In our pilot study, FRAK surgery induced a significant improvement of both UDVA and CDVA in patients with advanced keratoconus, including even those with a stage IV disease. Specifically, CDVA improved in 92.86\% of cases, with an improvement of more than 3 lines in 85.7\%. This visual improvement was associated to a significant flattening of the cornea and reduction of the magnitude of astigmatism. Future studies should evaluate the impact of the surgical procedure on corneal aberrations. Cunha et al. [27] reported the visual outcomes in 15 keratoconus patients after manual ipsilateral rotational penetrating autokeratoplasty technique (IRPAK) associated with a crescent-shape resection of $0.5 \mathrm{~mm}$ of the inferior cornea. They also found a reduction of topographic astigmatism and maximum and mean corneal curvature associated to visual improvement but of less magnitude than in our series [27]. Possibly, the generation of a more accurate and smooth corneal dissection with the femtosecond laser is the reason for obtaining a better visual outcome with our technique, as 
has been demonstrated with other applications of this laser technology [29]. Furthermore, no complications were detected during the surgical procedure and in the early postoperative period with our technique. Its non-penetrating nature helps to avoid potential serious complications which might occur when the ocular globe is completely opened. No reduction in endothelial cell count was observed, as happened in the study of Cunha et al. evaluating the outcomes of IRPAK [27].

We propose a new surgical technique that may be an alternative treatment option in advanced non-progressive keratoconus, allowing saving the patient's cornea instead of cornea transplantation. The potential advantages of the FRAK are high accuracy and reproducibility, an individual approach to surgical planning, a clinically relevant visual improvement, corneal regularization and flattening. A limitation of this study is the small number of participants and short follow-up, but the encouraging visual results could suggest the use of this technique in the assessment of its efficacy in larger series and evaluate the long-term results.

\section{Financial Disclosure}

None.

\section{References}

[1] Rabinowitz, Y.S. (1998) Keratoconus. Survey of Ophthalmology, 42, 297-319. http://dx.doi.org/10.1016/S0039-6257(97)00119-7

[2] Abu-Amero, K., Al-Muammar, A.M. and Kondkar, A.A. (2014) Genetics of Keratoconus: Where Do We Stand? Journal of Ophthalmology, 2014, Article ID 641708. http://dx.doi.org/10.1155/2014/641708

[3] Slonimsky, Y.B. and Gerasimov, A.S. (1992) Refractive Penetrating Corneal Transplantation. Novella, Moscow.

[4] Gore, D.M., Shortt, A.J. and Allan, B.D. (2013) New Clinical Pathways for Keratoconus. Eye, 27, 329-339. http://dx.doi.org/10.1038/eye.2012.257

[5] Shetty, R., Kaweri, L., Pahuja, N., Nagaraja, H., Wadia, K., Jayadev, C., Nuijts, R. and Arora, V. (2015) Current Review and a Simplified "Five-Point Management Algorithm” for Keratoconus. Indian Journal of Ophthalmology, 63, 46-53. http://dx.doi.org/10.4103/0301-4738.151468

[6] Alió, J.L. and Shabayek, M.H. (2006) Corneal Higher Order Aberrations: A Method to Grade Keratoconus. Journal of Refractive Surgery, 22, 539-545.

[7] Parker, J.S., van Dijk, K. and Melles, G.R. (2015) Treatment Options for Advanced Keratoconus. Survey of Ophthalmology, 60, 459-480. http://dx.doi.org/10.1016/j.survophthal.2015.02.004

[8] Kymionis, G.D. (2014) Long-Term Follow-Up of Corneal Collagen Cross-Linking for Keratoconus-The Cretan Study. Cornea, 33, 1071-1079. http://dx.doi.org/10.1097/ICO.0000000000000248

[9] Ziaei, M., Barsam, A., Shamie, N., Vroman, D., Kim, T., Donnenfeld, E., Holland, E., Kanellopoulos, J., Mah, F., Randleman, B., Daya, S. and Guell, J. (2015) Reshaping Procedures for the Surgical Management of Corneal Ectasia. Journal of Cataract \& Refractive Surgery, 41, 842-872. http://dx.doi.org/10.1016/j.jcrs.2015.03.010

[10] Vega-Estrada, A., Alio, J.L., Brenner, L.F., Javaloy, J., Plaza Puche, A.B., Barraquer, R.I., Teus, M.A., Murta, J., Henriques, J. and Uceda-Montanes, A. (2013) Outcome Analysis of Intracorneal Ring Segments for Keratoconus Based on Visual, Refractive, and Aberrometric Impairment. American Journal of Ophthalmology, 155, 575-584. http://dx.doi.org/10.1016/j.ajo.2012.08.020

[11] Slonimsky, Y.B., Slonimskiy, A.Y. and Korchuganova, Y.A. (2014) On the Issue of Rational Patient Management with Acute Keratoconus. Ophthalmology, 11, 17-24.

[12] Shehadeh-Mashor, R., Chan, C.C., Bahar, I., Lichtinger, A., Yeung, S. and Rootman, D. (2014) Comparison between Femtosecond Laser Mushroom Configuration and Manual Trephine Straight-Edge Configuration Deep Anterior Lamellar Keratoplasty. British Journal of Ophthalmology, 98, 35-39. http://dx.doi.org/10.1136/bjophthalmol-2013-303737

[13] Rama, P., Knutsson, K., Razzoli, G., Matuska, S., Viganò, M. and Paganoni, G. (2013) Deep Anterior Lamellar Keratoplasty Using an Original Manual Technique. British Journal of Ophthalmology, 97, 23-27. http://dx.doi.org/10.1136/bjophthalmol-2011-301168

[14] Kelly, T.L., Williams, K.A. and Coster, D.J., Australian Corneal Graft Registry (2011) Corneal Transplantation for Keratoconus: A Registry Study. Archives of Ophthalmology, 129, 691-697. http://dx.doi.org/10.1001/archophthalmol.2011.7

[15] Kelly, T.L., Coster, D.J. and Williams, K.A. (2011) Repeat Penetrating Corneal Transplantation in Patients with Keratoconus. Ophthalmology, 118, 1538-1542. http://dx.doi.org/10.1016/j.ophtha.2011.01.002 
[16] Kostenev, S.V. and Chernykh, V.V. (2012) Femtosecond Laser Surgery: Principles and Ophthalmological Application. Nauka (Science), Novosibirsk.

[17] Wetterstrand, O., Holopainen, J.M. and Krootila, K. (2015) Femtosecond Laser-Assisted Intrastromal Relaxing Incisions after Penetrating Keratoplasty: Effect of Incision Depth. Journal of Refractive Surgery, 31, 474-479. http://dx.doi.org/10.3928/1081597X-20150623-06

[18] Kopani, K.R., Page, M.A., Holiman, J., Parodi, A., Iliakis, B. and Chamberlain, W. (2014) Femtosecond Laser-Assisted Keratoplasty: Full and Partial-Thickness Cut Wound Strength and Endothelial Cell Loss across a Variety of Wound Patterns. British Journal of Ophthalmology, 98, 894-899. http://dx.doi.org/10.1136/bjophthalmol-2013-304546

[19] Maliugin, B.E., Pashtaev, A.N., Yelakov, Y.N., Kustova, K.I. and Aiba, E.E. (2012) Deep Anterior Lamellar Keratoplasty with the Use of Femtosecond Laser IntraLase $60 \mathrm{kHz}$; First Experience. Prakticheskaia Medicina (Practical Medicine), 4, 100-103.

[20] Arnalich-Montiel, F., Alió Del Barrio, J.L. and Alió, J.L. (2016) Corneal Surgery in Keratoconus: Which Type, Which Technique, Which Outcomes? Eye and Vision, 3, 2. http://dx.doi.org/10.1186/s40662-016-0033-y

[21] Günes, A., Kansu Bozkurt, T., Unlu, C., Sezgin Akcay, B.I. and Bayramlar, H. (2012) Ipsilateral Rotational Autokeratoplasty for the Management of Traumatic Corneal Scar. Case Reports in Ophthalmological Medicine, 2012, Article ID: 853584. http://dx.doi.org/10.1155/2012/853584

[22] Sharma, N., Sachdev, R., Titiyal, J.S., Tandon, R. and Vajpayee, R.B. (2012) Penetrating Autokeratoplasty for Unilateral Corneal Opacification. Eye \& Contact Lens, 38, 112-115. http://dx.doi.org/10.1097/ICL.0b013e318243e82c

[23] Murthy, S., Bansal, A.K., Sridhar, M.S. and Rao, G.N. (2001) Ipsilateral Rotational Autokeratoplasty: An Alternative to Penetrating Keratoplasty in Nonprogressive Central Corneal Scars. Cornea, 20, 455-457. http://dx.doi.org/10.1097/00003226-200107000-00002

[24] Arnalich-Montiel, F. and Dart, J.K. (2009) Ipsilateral Rotational Autokeratoplasty: A Review. Eye, 23, 1931-1938. http://dx.doi.org/10.1038/eye.2008.386

[25] Arora, R., Jain, P., Goyal, J.L. and Gupta, D. (2013) Comparative Analysis of Refractive and Topographic Changes in Early and Advanced Keratoconic Eyes Undergoing Corneal Collagen Crosslinking. Cornea, 32, 1359-1364. http://dx.doi.org/10.1097/ICO.0b013e3182a02ddb

[26] Ozertürk, Y., Sari, E.S., Kubaloglu, A., Koytak, A., Piñero, D. and Akyol, S. (2012) Comparison of Deep Anterior Lamellar Keratoplasty and Intrastromal Corneal Ring Segment Implantation in Advanced Keratoconus. Journal of Cataract \& Refractive Surgery, 38, 324-332. http://dx.doi.org/10.1016/j.jcrs.2011.08.033

[27] Cunha, F.J., Lira, W. and Brandt, C.T. (2011) Refractive and Topographic Changes of Ipsilateral Rotational Autokeratoplasty with a Crescent-Shape $0.5 \mathrm{~mm}$ Resection of the Inferior Cornea in Keratoconus after a 24 Months Follow-Up. Arquivos Brasileiros de Oftalmologia, 74, 184-189. http://dx.doi.org/10.1590/S0004-27492011000300008

[28] Cunha, F.J., Cavalcanti, M.T., Escarião, A.C., Arraes, T.A., Lira, W. and Cavalcanti, R. (2008) Ipsilateral Rotational Autokeratoplasty with $0.5 \mathrm{~mm}$ Resection of Inferior Corneal Crescent, in Keratoconus. Arquivos Brasileiros de Oftalmologia, 71, 410-414. http://dx.doi.org/10.1590/S0004-27492008000300020

[29] Piñero, D.P., Alio, J.L., El Kady, B., Coskunseven, E., Morbelli, H., Uceda-Montanes, A., Maldonado, M.J., Cuevas, D. and Pascual, I. (2009) Refractive and Aberrometric Outcomes of Intracorneal Ring Segments for Keratoconus: Mechanical versus Femtosecond-Assisted Procedures. Ophthalmology, 116, 1675-1687.

http://dx.doi.org/10.1016/j.ophtha.2009.05.016 